\title{
Slit-Robo signaling induces malignant transformation through Hakai-mediated E-cadherin degradation during colorectal epithelial cell carcinogenesis
}

Wei-Jie Zhou ${ }^{1, *}$, Zhen H Geng, ${ }^{2,}$, Shan Chi ${ }^{1, *}$, Wenli Zhang ${ }^{3, *}$, Xiao-Feng Niu ${ }^{1, *}$, Shu-Jue Lan ${ }^{1, *}$, Li Ma ${ }^{2}$, Xuesong Yang ${ }^{4}$, Li-Jing Wang ${ }^{5}$, Yan-Qing Ding ${ }^{3}$, Jian-Guo Geng $^{2,5}$

${ }^{1}$ Laboratory of Molecular Cell Biology, Institute of Biochemistry and Cell Biology, Shanghai Institutes for Biological Sciences, Chinese Academy of Sciences, 320 Yue-Yang Road, Shanghai 200031, China; ${ }^{2}$ Department of Biologic and Materials Sciences, University of Michigan School of Dentistry, Ann Arbor, MI 48109, USA; ${ }^{3}$ Department of Pathology, Nanfang Hospital and School of Basic Medical Sciences, Southern Medical University, Guangzhou, Guangdong 510515, China; ${ }^{4}$ Key Laboratory for Regenerative Medicine of the Ministry of Education, Medical College, Jinan University, Guangzhou, Guangdong 510632, China; ${ }^{5}$ Vascular Biology Research Institute, Guangdong Pharmaceutical University, Guangzhou, Guangdong 510006, China

Cell Research (2011) 21:992. doi:10.1038/cr.2011.94; published online 2 June 2011

Correction to: Cell Research (2011) 21:609-626. doi:10.1038/cr.2011.17; published online 1 February 2011

The authors apologize for the misplacement of the upper right panel of Figure 1G. The correct panel of Figure 1G is as follows.

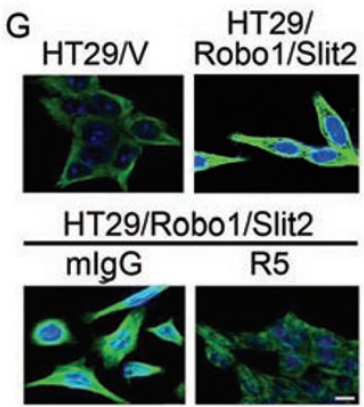

